\title{
“Silent" Acute Native Valve Leaflet Avulsion during Transcatheter Aortic Valve Replacement
}

\section{Abstract}

It is estimated that more than $8 \%$ of the population above the age of 85 suffer from significant aortic stenosis [1]. Transcatheter aortic valve replacement (TAVR) is increasing being utilized in the treatment of surgically high risk or inoperable patients with severe symptomatic aortic stenosis and has been shown to increase survival [2]. With the unavoidable operator learning curve, unexpected adverse events are likely to be encountered. These should be promptly recognized and the appropriate management strategy instituted. Feared complications include, annulus rupture, "frozen leaflet, coronary occlusion, embolization of the prosthetic device, Cerebrovascular accidents, as well as complications related to the vascular access site $[3,4]$. However, other uncommon events may occur that also have the potential to be catastrophic. We describe a case of acute native leaflet avulsion during a TAVR procedure. Fortunately, this was of no significant hemodynamic importance and the patient was successfully managed conservative.

Keywords: Aortic Stenosis; Transcatheter Aortic Valve Replacement; Percutaneous Artic Valve Replacement; Complications

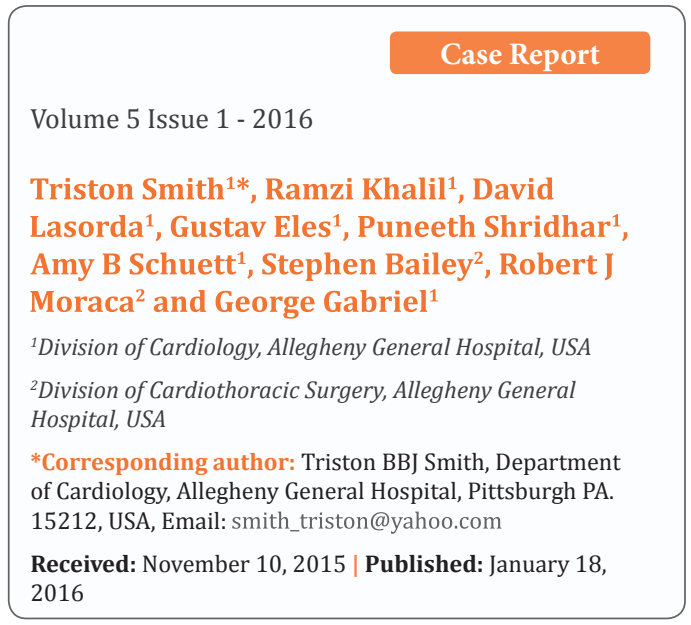

\section{Case Report}

An 86 year old female was referred to our facility for management of severe symptomatic aortic stenosis manifested as dyspnea at rest and syncope. Our multidisciplinary heart valve team deemed her to be high risk for surgical AVR due to NYHA functional class IV congestive heart failure, severe right ventricular dysfunction, pulmonary hypertension, chronic lung disease and diabetes mellitus. Transesophageal echocardiography (TEE) showed a trileaflet aortic valve with moderate to severe concentric leaflet calcification. The valve area was $0.4 \mathrm{~cm}^{2}$, with peak and mean gradients of 68 and $44 \mathrm{~mm} \mathrm{Hg}$ respectively. The left ventricular ejection fraction was 35\%. The patient's STS (Society of Thoracic Surgeons) risk of mortality score was 11 percent. On multi-detector computed tomography, the average basal ring diameter was determined to be $21 \mathrm{~mm}$. The transfemoral route was chosen because non-invasive assessment demonstrated suitable access site and vascular anatomy.

Vascular access was obtained via right common femoral artery cut down under general anesthesia. The patient was on dual antiplatelet therapy, DAPT (Clopidogrel and Aspirin), prior to the procedure and intra procedural anticoagulation with a total of $11,000 \mathrm{U}$ of intravenous unfractionated heparin was administered to maintain an ACT between 250 and 350. After the stenotic valve was crossed with an Amplatz Left 1 catheter (AL1) and a Newton wire, a guide wire exchange technique was used to position an Amplatz super stiff wire into the left ventricle. Aortic valvuloplasty was then performed using a $20 \mathrm{~mm}$ Edwards balloon. A $23 \mathrm{~mm}$ Edwards Sapiens (Edwards Life sciences, Irvine, CA) aortic valve was deployed, under rapid pacing of 180 beats/min (Figure 1A), using the Nova Flex+ transfemoral delivery system.

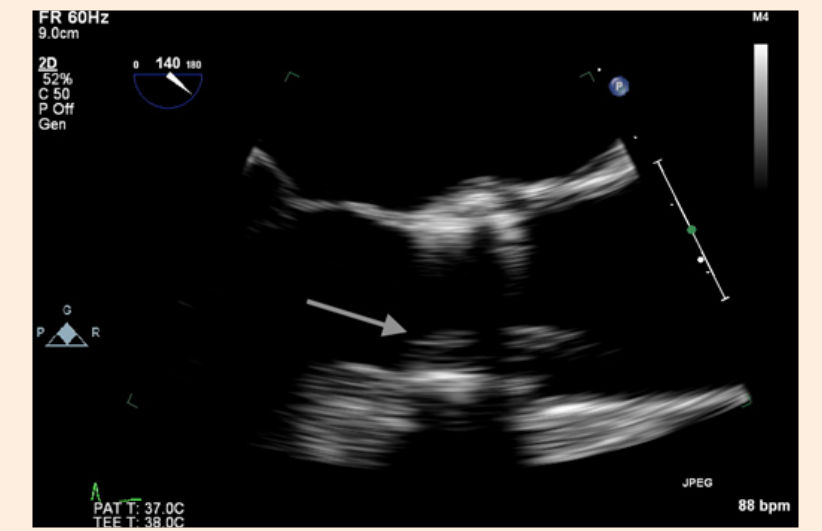

Figure 1A: Intraoperative TEE post valve deployment showing the echodensity prolapsing across the valve prosthesis into the aorta.

Immediately after the prosthetic valve deployment, intraprocedural TEE revealed a 20 x $9.5 \mathrm{~mm}$ mobile echo density attached to the ventricular side of the strut along the right coronary cusp. The structure intermittently prolapsed through the leaflets of the newly placed valve prosthesis during systole (Figure 1A, 1B). However, only mild $(1+)$ central aortic regurgitation was noted (Figure 2). There was no left ventricular outflow tract obstruction and the prosthetic valve leaflet excursion was otherwise normal. The peak and mean aortic valve gradients were 7 and $4 \mathrm{~mm} \mathrm{Hg}$ respectively post deployment.

There was a debate whether the echo density represented acute valve thrombosis vs an avulsed native valve leaflet. We believed the former to be less likely given the patient's pre 
treatment with DAPT and objective evidence of adequate intraprocedural anticoagulation. However, the patient was transferred to the intensive care unit for continuous monitoring. Anticoagulation with warfarin was initiated. Her hospital course was uneventful and she was discharged to home after 5 days. There was no change in the size of the echo density or severity of aortic regurgitation on pre-discharge TEE. The decision was made to treat the patient with 3 months of anticoagulation with DAPT and warfarin. Follow up TEE at 3 months revealed a persistent mobile echo density, unchanged in appearance from the intraprocedural TEE, confirmed by 3D echo cardio graphic reconstruction (Figure 3 ). The patient continued to be symptom free and was advised to remain on daily aspirin and warfarin. Clopidogrel was discontinued.

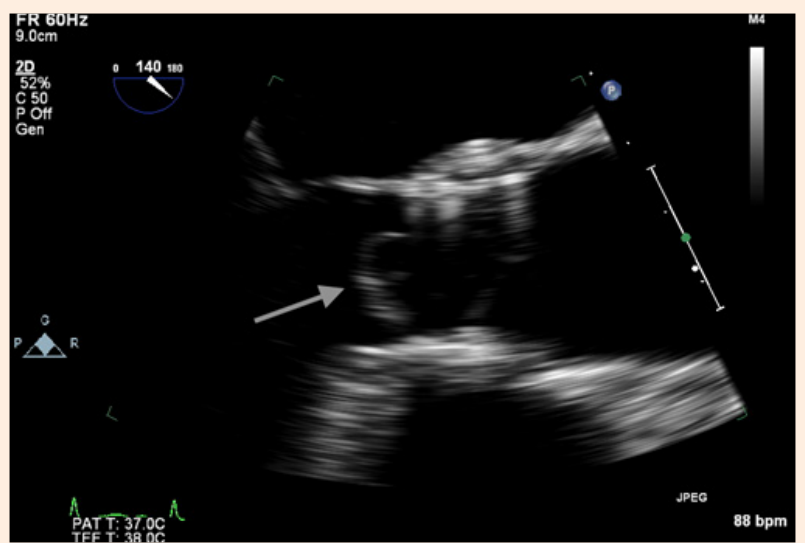

Figure 1B: Intraoperative TEE post valve deployment showing an echodensity attached to the ventricular side of the strut along the right coronary cusp protruding into the left ventricular outflow tract.

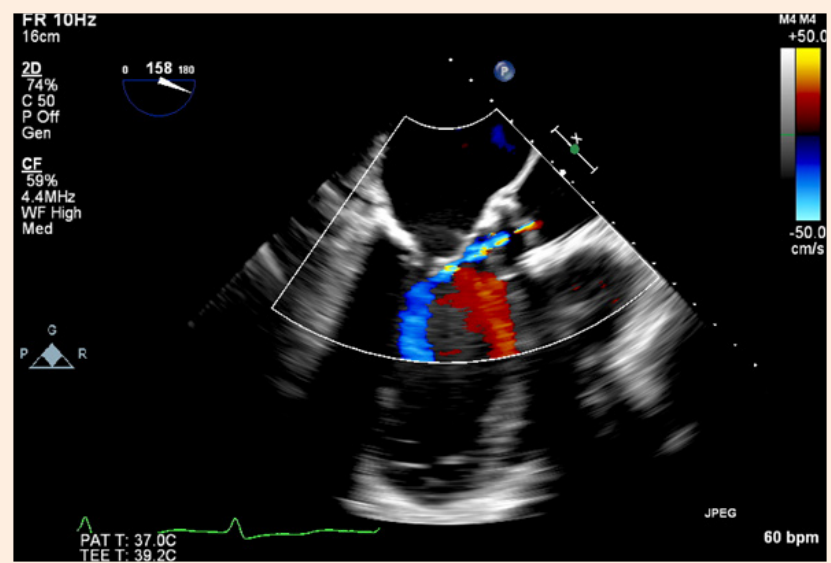

Figure 2: Intraoperative TEE post deployment showing mild central aortic insufficiency

"Silent" acute avulsion of an aortic valve leaflet without annulus rupture might represent a rare complication of TAVR. Our review of the current literature did not unearth any published case series or observational studies, so we are unable to quantify its incidence. The differential diagnosis of an acute mobile echo density during TAVR includes valve thrombosis versus the remnant of native valve tissue. Thrombosis of both the Core valve and the Edward Sapien transcatheter valve, although rare, have been documented [5]. Acute valve thrombosis can be fatal and it is extremely important to rule out this diagnosis. Although we were not convinced that the echo density represented thrombus, our team decided to treat the patient with anticoagulation as a precautionary measure due to the potential catastrophic implications. Follow up TEE confirmed our initial diagnosis.

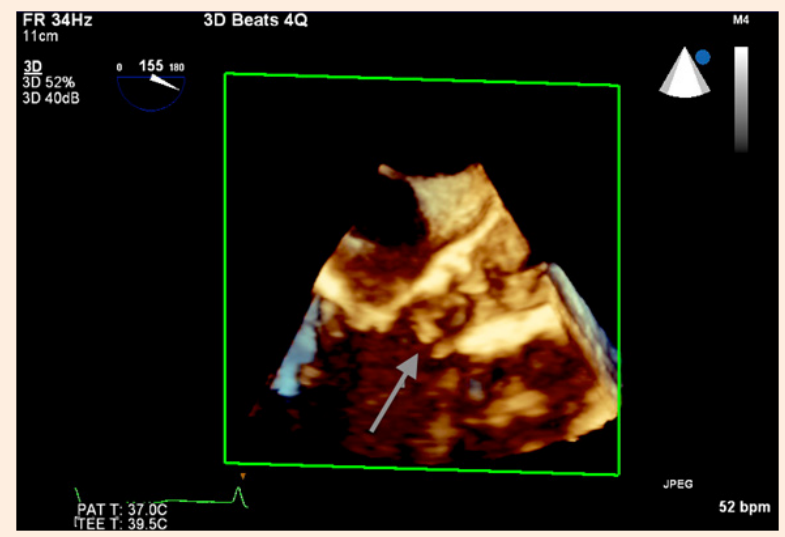

Figure 3: 3D reconstructed image 3 months post op showing the persistent mobile mass

In our case, the echogenicity was believed to be secondary to the native leaflet avulsion since its characteristics remained unchanged on serial echocardiograms. The most likely reason for the prolapse of the native leaflet might have been the tight fit of the delivery system although this cannot be confirmed. A similar entity has been recently reported by Zimmet et al. [6]. However, aortic annulus rupture was also present and the partially avulsed leaflet resulted in hemodynamic instability requiring further treatment [4]. In our case, the patient remained stable and was asymptomatic.

The long-term implications of a "silent" avulsed aortic leaflet in the presence of percutaneously inserted aortic valve prosthesis is currently unknown. The question regarding whether this complication requires long term anticoagulation, especially in this group of patients with elevated frailty scores and high fall risk also needs to be explored.

\section{References}

1. Leibowitz D, Stessman J, Jacobs JM, Stessman-Lande I, Gilon D (2013) Prevalence and prognosis of aortic valve disease in subjects older than 85 years of age. Am J Cardiol 112(3): 395-399.

2. Svensson LG, Blackstone EH, Rajeswaran J, Brozzi N, Leon MB, et al. (2014) Comprehensive analysis of mortality among patients undergoing TAVR: results of the PARTNER trial. J Am Coll Cardiol 64(2): 158-168.

3. Kapadia SR, Svensson LG, Roselli E, Schoenhagen P, Popovic Z, et al. (2014) Single center TAVR experience with a focus on the prevention and management of catastrophic complications. Catheter Cardiovasc Interv 84(5): 834-842.

4. Agostoni P, Buijsrogge MP, Stella PR (2012) "Frozen" leaflet: a dreadful complication of transcatheter aortic valve implantation. Circ Cardiovasc Interv 5(2): 321-323. 
5. Sanon S, Maleszewski JJ, Rihal CS (2014) Hydrophilic polymer embolism induced acute transcatheter aortic valve thrombosis: a novel complication. Catheter Cardiovasc Interv 83(7): 1152-1155.
6. Zimmet J, Kaiser E, Tseng E, Shunk K (2014) Successful Percutaneous Management of Partial Avulsion of the Native Aortic Valve Complex Complicating Transcatheter Aortic Valve Replacement. J Invasive Cardiol 26(10): 137-140. 